\title{
Natural resonance frequency of the brain depends on only intracranial pressure: clinical research
}

\author{
Tetsuya Goto ${ }^{1,2}$, Kenji Furihata ${ }^{1 *} \&$ Kazuhiro Hongo ${ }^{1}$ \\ To understand and control intracranial pressure (ICP) is required for treatments in various clinical \\ situations. To establish non-invasive ICP prediction method, we focused on the natural resonance \\ frequency (NRF) of the brain. The ICP value, pulse waveform of intracranial pressure (PWICP) and \\ cervical carotid pulse waveform (CCPW) were simultaneously collected from patients who underwent \\ neurosurgical treatment. A total of 43 data were obtained from 27 patients. The total measured time \\ was 29,653 seconds and the measured mean ICP value in each data ranged from 3.82 to 69.39 (mean \\ 25.9) $\mathrm{hPa}$. Respiratory synchronized cardiac pulses were selected and following CCPW and PWICP \\ were collected. The transfer characteristics from collected CCPW to PWICP were calculated. The initial \\ negative peak was judged as the NRF of the brain. The relationship between the ICP value and the \\ NRF of the brain was presented on the quadratic functions graph $\left(I C P=0.0329(N R F)^{2}+0.0842 N R F\right.$; \\ $\left.R^{2}=0.9952\right)$. It means that the individual NRF only depends on their ICP value. The ICP value will be \\ predicted by checking NRF of the brain from somewhere.
}

The need to understand and control intracranial pressure (ICP) is required for treatments in clinical neurosurgery, neurology and emergency medicine. The ICP value can only be measured by direct intracranial catheter placement, for example, through ventricular drainage or an ICP sensor ${ }^{1-3}$. Transcranial Doppler ultrasonography ${ }^{4}$, tympanic membrane displacement ${ }^{5,6}$, and optic nerve sheath diameter ${ }^{7}$ have been investigated for non-invasive ICP monitoring, A preliminary report of the HS-1000 (HeadSense Medical Ltd.), in which the HS-1000 evaluated an acoustic signal from the ipsilateral ear including a short beep from the contralateral ear, has possibility of non-invasive ICP monitoring ${ }^{8}$. On the other hand, Raboel et al. stated that they were inaccurate and could not be used as an alternative to invasive techniques ${ }^{9-11}$. Recent well-designed research also presented that the ICP could not be predicted by tympanic membrane pressure ${ }^{6}$.

Our motivation for this research is to predict the ICP value by analysing the extra-auditory canal pressure waveform (EACPW). It is considered that the EACPW includes the component of ICP value because of their anatomical connection ${ }^{5,6,12-16}$. We theorised that the ICP value could be predicted with the use of the transfer function method from the cervical cardiac pulse waveform (CCPW) to EACPW ${ }^{12}$. To predict the ICP value from EACPW, for which there is no information of the direct current components included, we focused on the natural resonance frequency (NRF) of the brain. The particular structure has its own NRF, which depends on its consistency. The brain is in an individual NRF and the NRF correlates with the ICP; resonance phenomenon of the brain can be observed with the bump of the arterial pulse in every cardiac pulse. Ommaya et al. observed low-range natural frequencies $(5-10 \mathrm{~Hz})$ in the rotational brain motion of primates and humans in $1960 \mathrm{era}^{17}$. System transfer function analysis of intracranial cavity has been studied in animal model since $1980 \mathrm{era}^{18-21}$. By extent information about NRF of the brain, idea of non-invasive ICP prediction which was based on human brain tissue resonance frequency correlation with ICP value has been proposed by Michaeli D. et al. ${ }^{22}$. In recent papers $^{23,24}$, although it is considered that the NRF of the brain is determined by the brain weight and CSF volume compliance, the theory was based on the MRI findings. The pulse waveform of intracranial pressure (PWICP) and CCPW have not been analyzed in individual clinical patients.

We discovered a simple relationship between the ICP value and the NRF of the brain by the transfer function method using the CCPW and PWICP from our clinical data. By utilising this algorithm, the individual ICP value can be predicted by a patient's NRF. Our results and the mechanism of the relationship between the ICP value and the NRF of the brain are discussed in this report.

${ }^{1}$ Department of Neurosurgery, Shinshu University School of Medicine, Matsumoto, Japan. ${ }^{2}$ Department of Neurosurgery, St. Marianna University School of Medicine, Kawasaki, Japan. *email: kennfur@shinshu-u.ac.jp 


\begin{tabular}{|c|c|c|c|c|c|c|c|}
\hline \multicolumn{8}{|c|}{ Materials } \\
\hline Patient & $\begin{array}{l}\text { Age } \\
\text { (year) }\end{array}$ & Sex & \begin{tabular}{|l|} 
Body \\
height (m)
\end{tabular} & $\begin{array}{l}\text { Body weight } \\
\text { (kg) }\end{array}$ & Diagnosis & ICP measurement & \begin{tabular}{|l|} 
Data \\
number
\end{tabular} \\
\hline 1 & 42 & $\mathrm{w}$ & 1.65 & 41 & glioma & drainage & 1 \\
\hline \multirow{2}{*}{2} & \multirow{2}{*}{51} & \multirow{2}{*}{ w } & \multirow{2}{*}{1.58} & \multirow{2}{*}{58} & \multirow{2}{*}{ glioma } & \multirow{2}{*}{ drainage } & $2 \_1$ \\
\hline & & & & & & & $2 \_2$ \\
\hline \multirow{2}{*}{3} & \multirow{2}{*}{52} & \multirow{2}{*}{$\mathrm{m}$} & \multirow{2}{*}{1.58} & \multirow{2}{*}{62} & \multirow{2}{*}{ MCA occlusion } & \multirow{2}{*}{ drainage } & $3 \_1$ \\
\hline & & & & & & & $3 \_2$ \\
\hline \multirow{2}{*}{4} & \multirow{2}{*}{49} & \multirow{2}{*}{ w } & \multirow{2}{*}{1.52} & \multirow{2}{*}{59} & \multirow{2}{*}{ meningioma } & \multirow{2}{*}{ drainage } & $4 \_1$ \\
\hline & & & & & & & $4 \_2$ \\
\hline 5 & 67 & $\mathrm{~m}$ & 1.65 & 60 & hydrocephalus & drainage & 5 \\
\hline \multirow{3}{*}{6} & & & & & & & $6 \_1$ \\
\hline & 29 & $\mathrm{~m}$ & 1.7 & 57 & hydrocephalus & drainage & $6 \_2$ \\
\hline & & & & & & & $6 \_3$ \\
\hline & & & & & & & 7_1 \\
\hline 7 & 23 & $\mathrm{~m}$ & 165 & 63 & ASDH & ICP sensor enidur & 7_2 \\
\hline 1 & 23 & $\mathrm{~m}$ & 1.65 & 63 & ASDH & ICP sensor epiaura & $7 \_3$ \\
\hline & & & & & & & 7_4 \\
\hline & & & & & & & $8 \_1$ \\
\hline 8 & 47 & $\mathrm{~m}$ & 1.75 & 66 & $\mathrm{ICH}$ & ICP sensor brain & $8 \_2$ \\
\hline & & & & & & & $8 \_3$ \\
\hline 9 & 55 & $\mathrm{~m}$ & 1.7 & 103 & brain contusion & drainage & 9 \\
\hline 10 & 53 & $\mathrm{~m}$ & 1.69 & 77 & vestibular schwannoma & drainage & 10 \\
\hline 11 & 42 & $\mathrm{~m}$ & 1.87 & 74 & hydrocephalus & drainage & 11 \\
\hline 12 & 62 & $\mathrm{w}$ & 1.56 & 49 & ICH & drainage & 12 \\
\hline 13 & 14 & $\mathrm{~m}$ & 1.71 & 55 & glioma & drainage & 13 \\
\hline 14 & 75 & $\mathrm{w}$ & 1.55 & 50 & ASDH & ICP sensor epidura & 14 \\
\hline 15 & 62 & $\mathrm{w}$ & 1.37 & 32 & hydrocephalus & drainage & 15 \\
\hline & & & & & & & $16 \_1$ \\
\hline 16 & 54 & $\mathrm{~m}$ & 172 & 71 & brain contusion & ICP sensor enidure & $16 \_2$ \\
\hline 10 & 54 & $\mathrm{~m}$ & $1 . / 2$ & $/ 1$ & Drain contusion & ICP sensor epiaura & $16 \_3$ \\
\hline & & & & & & & $16 \_4$ \\
\hline 17 & 77 & $\mathrm{w}$ & 1.42 & 61 & ASDH & drainase & $17 \_1$ \\
\hline 1) & 17 & w & 1.42 & 61 & ASDH & arainage & $17 \_2$ \\
\hline 18 & 20 & $\mathrm{w}$ & 1.63 & 67 & vestibular schwannoma & drainage & 18 \\
\hline 19 & 64 & $\mathrm{w}$ & 1.54 & 40 & meningitis & drainage & 19 \\
\hline & & & & & & & $20 \_1$ \\
\hline 20 & 69 & $\mathrm{~m}$ & 1.81 & 82 & MCA occlusion & drainage & $20 \_2$ \\
\hline & & & & & & & $20 \_3$ \\
\hline 21 & 77 & $\mathrm{~m}$ & 1.6 & 60 & MCA occlusion & drainage & 21 \\
\hline 22 & 83 & $\mathrm{w}$ & 1.4 & 39 & subarachnoid hemorrhage & drainage & 22 \\
\hline 23 & 68 & $\mathrm{~m}$ & 1.71 & 59 & meningioma & drainage & 23 \\
\hline 24 & 67 & $\mathrm{~m}$ & 1.64 & 53 & ICH & drainage & 24 \\
\hline 25 & 17 & $\mathrm{~m}$ & 1.73 & 61 & glioma & drainage & 25 \\
\hline 26 & 74 & $\mathrm{~m}$ & 1.68 & 77 & brain contusion & drainage & 26 \\
\hline 27 & 74 & $\mathrm{~m}$ & 1.67 & 63 & CSF leakage & drainage & 27 \\
\hline
\end{tabular}

Table 1. ICP: intracranial pressure, MCA: middle cerebral artery, ASDH: acute subdural hematoma, ICH: intracerebral hematoma.

\section{Materials and Methods}

Patient criteria. The ICP value, PWICP and CCPW were simultaneously collected from patients who had undergone ventricular drainage or ICP sensor placement for medical treatment at Shinshu University Hospital from November 2014 to March 2017. All experiments were performed in accordance with relevant guidelines and regulations. The measurement of this information was approved by the Ethics Committee of Shinshu University School of Medicine "Development of non-invasive intracranial pressure monitoring" approved number 2343. Informed consent was obtained from all the patients or their families. Some participants under the age of 18 years, informed consent has been obtained from a parent.

A total of 27 patients, 17 men and 10 women, were included in the present research. The age of the patients ranged from 14-83 (mean 54) years, body height of 137-187 (mean 163) cm and body weight 32-103 (mean 


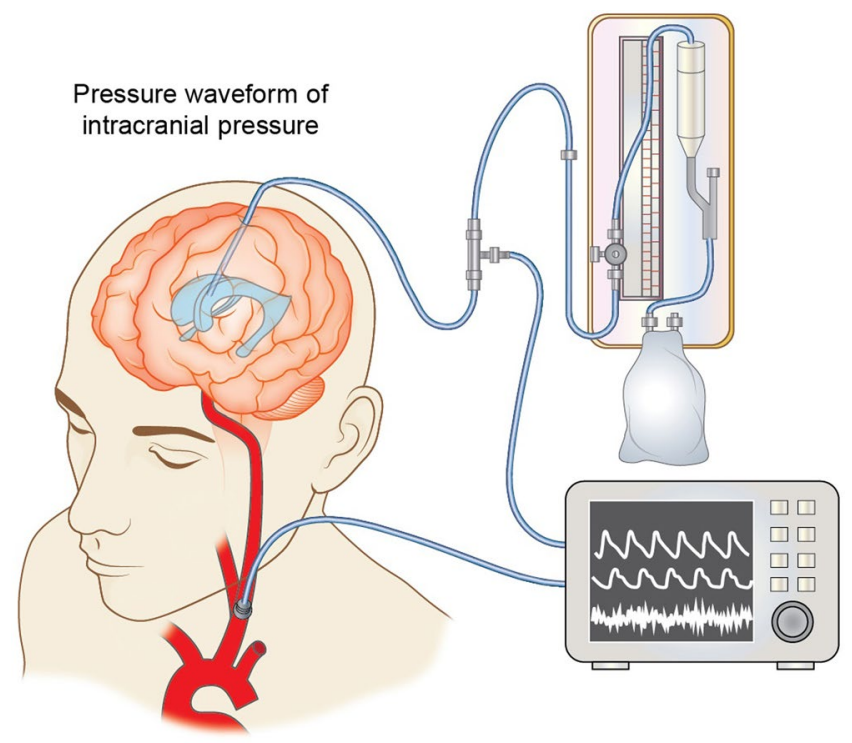

Cervical carotid pulse waveform

Figure 1. A conceptual schema of data sampling. Cervical carotid pulse waveform and pressure waveform of intracranial pressure were collected and stored simultaneously in $200 \mathrm{~Hz}$ by original device.

61) kg. Of the 27 patients six had a presentation of trauma, four had glioma, five had an extra-axial tumour, four had intracerebral haemorrhage, three had infarction, four had hydrocephalus and one had a cerebrospinal fluid leakage. The ICP was measured by an ICP sensor in four patients and ventricular drainage in 23 patients (Table 1).

Recording the data. The ICP value and PWICP were measured by either ventricular drainage or an ICP sensor. The ventricular drainage, in which $0 \mathrm{~cm} \mathrm{H}_{2} \mathrm{O}$ height was adjusted at the level of the extra-auditory canal, was connected to the pressure sensor (SCK-7604 transducer blood sampling set: Argon Medical Devices: Frisco TX, USA). The ICP sensor (Integra Camino ${ }^{\circledR}$ Intracranial Pressure Monitor: Integra LifeSciences Corporation: Plainsboro NJ, USA) was placed at the subdural space or intra-parenchymal portion. CCPW is pulse tonometry. Original sensor was made by approximately $2 * 10^{-6} \mathrm{~m}^{3}$ volume of polyethylene airtight cylinder. The cylinder, which base was flat and round $8 \mathrm{~mm}$ in diameter, was placed on the neck at the position of the cervical carotid artery and fitted by a soft band. A pressure change in the cylinder was measured by high sensitive microphone (EM215 Electric Condenser Microphone: Primo Corporation: Tokyo, Japan).

All the information was collected and stored simultaneously in $200 \mathrm{~Hz}$ by original device made by Ichikawa Electric Corp. Ltd. Tokyo, Japan (Fig. 1).

In some patients measurements were undertaken on different days as different data.

Analysing the data. The data was analysed in an off-line state. All noise as a result of patient motion, artifact, or inappropriate sensor setting were rejected before analysis. (1) After rejection of the noise, the consecutive data was cut into 10.24 seconds, which was called a "column". Each column covered a 5.12-second width. (2) The largest positive peak of cardiac pulsation of CCPW was picked-up from each column, as well as one synchronized PWICP. (3) The picked-up CCPW and PWICP were averaged using the weighted average method. (4) The transfer characteristics from CCPW to PWICP were calculated using the transfer function method, after pressure transmission to the CCPW by the substitution of systolic/diastolic blood pressure value from the upper arm (Fig. 2). (5) The NRF of the brain was calculated value as initial pole of the transfer characteristics. Figure 3 graph represented the relationship between transfer function magnitude and frequency. The NRF of the brain (yellow arrow) located near the first negative peak in the graph. (6) The ICP value was averaged in the whole of the selected columns, and the relationship between the ICP value and the NRF of the brain was compared.

Calculation of transfer characteristics between CCPW and PWICP. The relationship was verified by Matlab version 2018b (The Mathworks, Natick, MA, USA). Transfer function models described the relationship between the inputs and outputs of a system using a ratio of polynomials. The model order was equal to the order of the denominator polynomial. The roots of the denominator polynomial were referred to as the model poles. The roots of the numerator polynomial were referred to as the model zeros. The parameters of a transfer function model were its poles, zeros and transport delays.

Where, $P W I C P(s), C C P W(s)$ and noise (error: $E(s)$ ) represented the Laplace transforms of the output, input and noise, respectively. In continuous-time, a transfer function model had the form: 

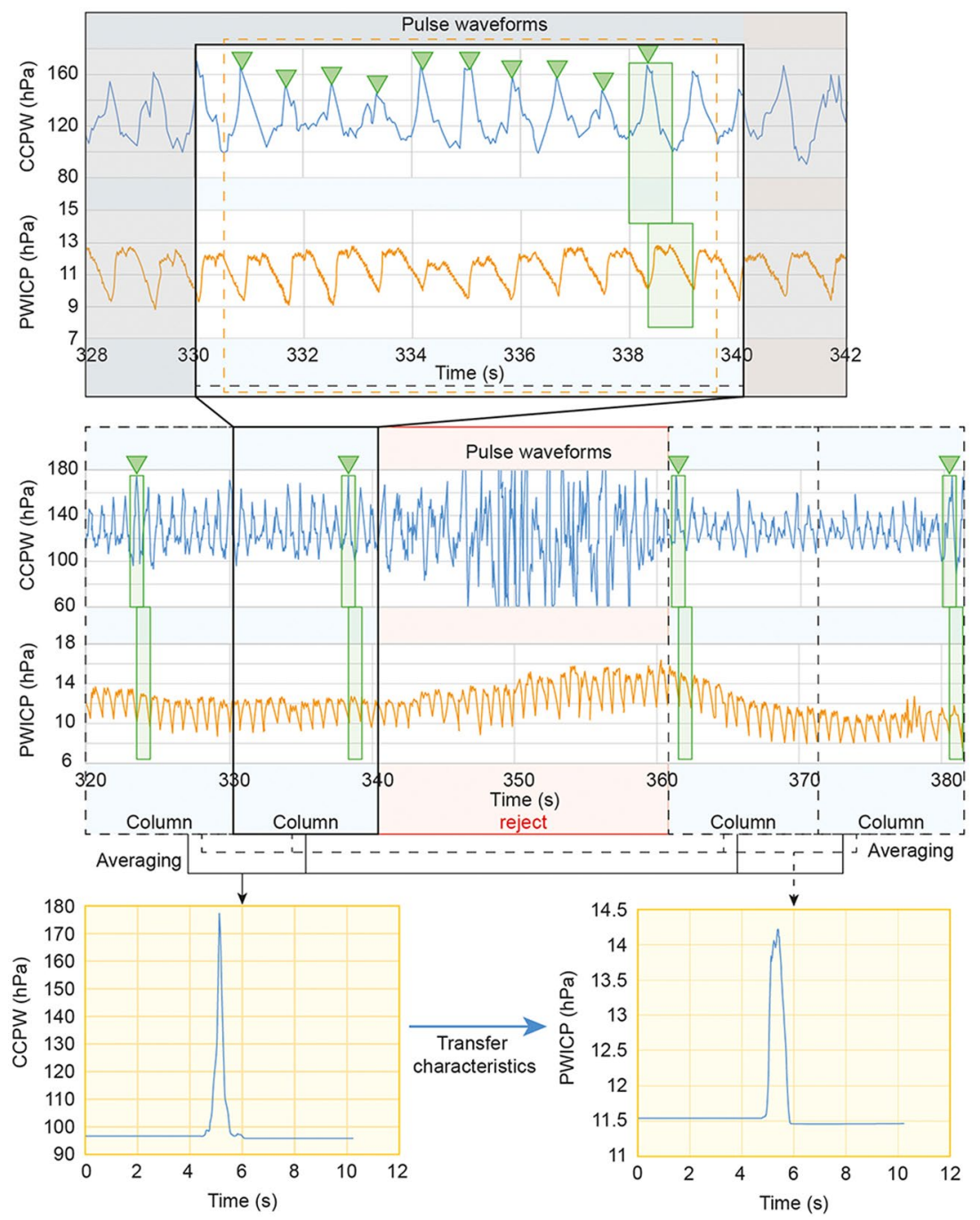

Figure 2. How to calculate the transfer characteristics between cervical carotid pressure waveform and pulse waveform of intracranial pressure. Center graph: The raw data of cervical carotid pulse waveform (CCPW) and the pulse waveform of intracranial pressure (PWICP). After rejection of the noise (red line square), the data was cut in consecutive 10.24 seconds which was called a "column" (black dot square). Upper graph: magnified one column. The largest one (green square) from the positive peak of cardiac pulsation of CCPW (green arrowhead) was selected in each column, as well as an synchronized PWICP. Lower two waveforms: The selected CCPW and PWICP were averaged by the weighted average method. Left panel: averaged CCPW after pressure transmission to the CCPW by the substitution of systolic/diastolic blood pressure value from the upper arm, Right panel: averaged PWICP. And the transfer characteristics from CCPW to PWICP was calculated.

$$
P W I C P(s)=\frac{n u m(s)}{\operatorname{den}(s)} e^{-s \tau} C C P W(s)+E(s) .
$$

Numerator: $n u m(s)$ and denominator: $\operatorname{den}(s)$ polynomials that defined the relationship between the input and the output. $\tau$ represented the delay. The choice of a model order was also affected by the amount of delay. The first step, a good idea of the input delay simplified the key task of matching up the rise points between the averaged CCPW and PWICP (See Figs. 2-3).

If we took the Z-transform, where, $P W I C P\left(z^{-1}\right), C C P W\left(z^{-1}\right)$ and $E\left(z^{-1}\right)$ represented the Z-transforms of the output, input and noise, respectively. $z^{-1}$ was the Z-transform of the lag operator. The transfer function had the form:

$$
\begin{gathered}
P W I C P\left(z^{-1}\right)=\frac{n u m\left(z^{-1}\right)}{\operatorname{den}\left(z^{-1}\right)} \operatorname{CCPW}\left(z^{-1}\right)+E\left(z^{-1}\right), \\
\operatorname{num}\left(z^{-1}\right)=b_{0}+b_{1} z^{-1}+b_{2} z^{-2}+b_{3} z^{-3}+\cdots+b_{n} z^{-n},
\end{gathered}
$$




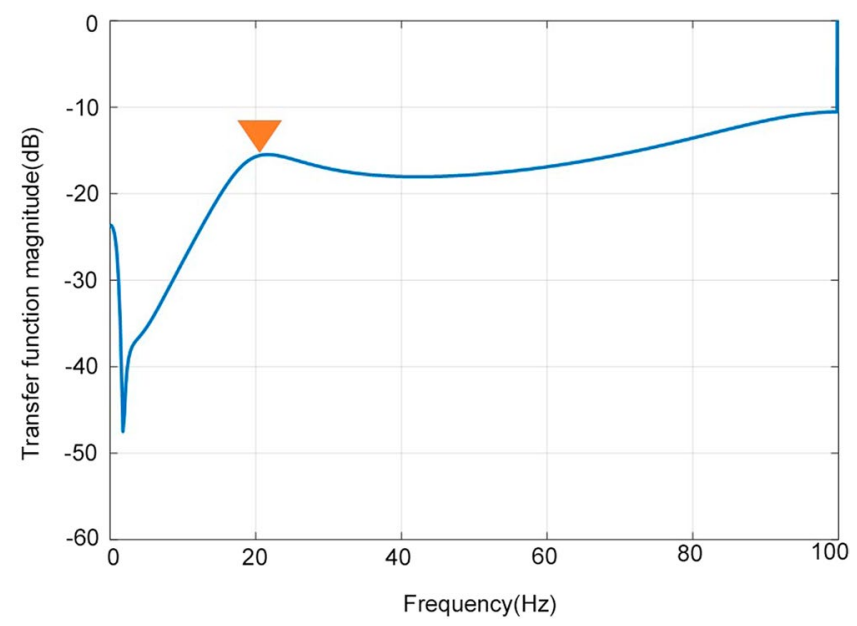

Figure 3. The graph for the relationship between transfer function magnitude and frequency. The yellow arrow indicates the NRF of the brain.

$$
\operatorname{den}\left(z^{-1}\right)=1+a_{1} z^{-1}+a_{2} z^{-2}+a_{3} z^{-3}+\cdots+a_{n} z^{-n}
$$

where, $\mathrm{np}$ was number of poles and $\mathrm{nz}$ was number of zeros in the estimated transfer function. Manually matching complicated features in the Bode plot (magnitude and phase data) of the frequency response of the high-order brain system was difficult to match with conventional first and second order numerators and denominators. The next task was to identify the orders. This method often required manual tuning of weighting parameters. Therefore, we checked the first resonance frequency, the Q (quality factor) and the fit for all 243 combinations of the np changed from three to eight, the $\mathrm{nz}$ changed from one to seven and the fine adjustment delays changed from zero to eight.

The fit showed that normalized root mean squared error (NRMSE) expressed as a percentage, defined as:

$$
\text { FitPercent }=100\left(1-\frac{\left\|P W I C P_{\text {measured }}-P W I C P_{\text {model }}\right\|}{\| P W I C P_{\text {measured }}-\overline{P W I C P_{\text {measured }} \|}}\right) .
$$

Where, $P$ WICP $P_{\text {measured }}$ was the measured PWICP data, $\overline{\text { PWICP }_{\text {measured }}}$ was its mean, $P W I C P_{\text {model }}$ was the simulated response of the model, and $\|\cdots\|$ indicated the 2-norm of a vector. FitPercent varies between -Inf (bad fit) to 100 (perfect fit). If the value is equal to zero, then the model is no better at fitting the measured data than a straight line equal to the mean of the data.

From the calculated results, three functions of model order could be determined by analysing the improvement in the first resonance frequency (nearest neighbour search of the theoretical NRF), the $\mathrm{Q}(0.5<\mathrm{Q}<10)^{25}$ and the fit (fit $>60 \%$ ).

Statistical analysis. We tried to model the obtained data using a second-degree polynomial function:

$$
I C P=c_{2} N R F^{2}+c_{1} N R F+c_{0} .
$$

The unknown coefficient, $c_{0}, c_{1}$ and $c_{2}$ were computed by minimizing the sum of the squares of the deviations of the data from the model (least squares fit). For the first step we used "polyfit" to find the polynomial coefficients. Matlab calculated the polynomial coefficients in descending powers. In the second step we used "polyval" to evaluate at the measured NRF. On the third step we identified the statistical determination between the variables ICP and $N R F$ to justify modelling the data. A data model explicitly described a relationship between predictor and response variables. Linear regression fitted a data model that was linear in the model coefficients. The most common type of linear regression was a least squares fit, which would fit both polynomials. One method to find the better fit was to calculate the coefficient of determination, $R^{2}$. $R^{2}$ was one measure of how well a model would predict the data and fell between zero and one. The higher the value of $R^{2}$, the better the model was at predicting data.

Where $\widehat{I C P}$ represented the calculated values of $I C P$ and $\overline{I C P}$ was the mean of $I C P . R^{2}$ was defined as:

$$
R^{2}=1-\frac{\sum_{i=1}^{n}\left(I C P_{i}-\widehat{I C P}\right)^{2}}{\sum_{i=1}^{n}\left(I C P_{i}-\overline{I C P}\right)^{2}} .
$$

Ethics. The simultaneous measurement of intracranial pressure and cervical carotid artery pressure waveform was approved by Ethical Committee of Shinshu University School of Medicine. "Development of non-invasive intracranial pressure monitoring" approved number 2343. https://www.shinshu-u.ac.jp/faculty/medicine/common/ docs/i-rinri/r-shounin25.pdf (in Japanese). 


\begin{tabular}{|c|c|c|c|c|c|c|c|}
\hline \multirow{3}{*}{$\begin{array}{l}\text { Data } \\
\text { number }\end{array}$} & \multicolumn{7}{|c|}{ Measured data } \\
\hline & $\begin{array}{l}\text { Measured } \\
\text { time }\end{array}$ & $\begin{array}{l}\text { Reject } \\
\text { time }\end{array}$ & $\begin{array}{l}\text { Valid } \\
\text { data } \\
\text { time }\end{array}$ & Number of columns & $\begin{array}{l}\text { ICP } \\
\text { value }\end{array}$ & \begin{tabular}{|l} 
ICP \\
value \\
STD
\end{tabular} & \begin{tabular}{|l|} 
Blood \\
pressure \\
(sistoric/ \\
diastoric)
\end{tabular} \\
\hline & (s) & (s) & (s) & $($ Column $=10.24 \mathrm{~s})$ & (hPa) & (hPa) & (hPa) \\
\hline 1 & 272 & 47 & 225 & 44 & 31.08 & 4.22 & $160 / 93$ \\
\hline $2 \_1$ & 319 & 19 & 300 & 60 & 9.66 & 1.57 & $170 / 93$ \\
\hline $2 \_2$ & 701 & 199 & 502 & 98 & 19.40 & 3.51 & $146 / 93$ \\
\hline $3 \_1$ & 1631 & 1529 & 102 & 20 & 35.56 & 12.01 & $239 / 81$ \\
\hline $3 \_2$ & 295 & 85 & 210 & 41 & 16.50 & 8.63 & $186 / 106$ \\
\hline $4 \_1$ & 697 & 259 & 438 & 85 & 13.60 & 1.08 & $181 / 100$ \\
\hline $4 \_2$ & 736 & 335 & 401 & 78 & 13.72 & 2.62 & $173 / 93$ \\
\hline 5 & 867 & 398 & 469 & 91 & 19.27 & 1.12 & $190 / 101$ \\
\hline $6 \_1$ & 2562 & 1905 & 657 & 128 & 36.69 & 4.50 & $173 / 88$ \\
\hline $6 \_2$ & 1183 & 349 & 834 & 163 & 40.17 & 8.52 & $180 / 100$ \\
\hline $6 \_3$ & 653 & 131 & 522 & 104 & 9.31 & 1.89 & $170 / 93$ \\
\hline 7_1 & 413 & 20 & 393 & 76 & 44.23 & 0.63 & $198 / 100$ \\
\hline 7_2 & 368 & 0 & 368 & 71 & 45.17 & 0.40 & $184 / 106$ \\
\hline 7_3 & 444 & 20 & 424 & 82 & 30.98 & 0.18 & $166 / 93$ \\
\hline 7_4 & 661 & 68 & 593 & 115 & 44.08 & 3.80 & $180 / 113$ \\
\hline 8 & 359 & 38 & 321 & 62 & 36.49 & 1.36 & $201 / 78$ \\
\hline $8 \_2$ & 333 & 10 & 323 & 63 & 37.41 & 1.63 & $216 / 81$ \\
\hline $8 \_3$ & 643 & 57 & 586 & 114 & 62.54 & 4.80 & $216 / 106$ \\
\hline 9 & 300 & 0 & 300 & 58 & 6.24 & 0.30 & $190 / 82$ \\
\hline 10 & 438 & 139 & 299 & 58 & 13.42 & \begin{tabular}{|l|l|}
0.92 \\
\end{tabular} & $186 / 126$ \\
\hline 11 & 671 & 168 & 503 & 98 & \begin{tabular}{|l|}
13.28 \\
\end{tabular} & 3.49 & $173 / 93$ \\
\hline 12 & 1292 & 803 & 489 & 134 & 10.57 & 3.64 & $160 / 80$ \\
\hline 13 & 856 & 432 & 424 & 82 & 21.57 & 2.52 & $146 / 67$ \\
\hline 14 & 949 & 77 & 872 & 170 & 69.39 & 1.22 & $186 / 93$ \\
\hline 15 & 957 & 348 & 609 & 119 & \begin{tabular}{|l|}
15.89 \\
\end{tabular} & 2.01 & $146 / 80$ \\
\hline $16 \_1$ & 1222 & 710 & 512 & 100 & 44.71 & \begin{tabular}{|l|}
0.94 \\
\end{tabular} & $173 / 93$ \\
\hline $16 \_2$ & 549 & 20 & 529 & 103 & 33.21 & \begin{tabular}{|l|} 
\\
\end{tabular} & $186 / 100$ \\
\hline $16 \_3$ & 472 & 336 & 136 & 26 & 46.31 & 0.55 & $173 / 88$ \\
\hline $16 \_4$ & 256 & 10 & 246 & 48 & 13.38 & \begin{tabular}{|l|l|}
0.19 \\
\end{tabular} & $160 / 100$ \\
\hline 17_1 & 733 & 0 & 733 & 143 & 21.66 & \begin{tabular}{|l|}
1.02 \\
\end{tabular} & $193 / 82$ \\
\hline $17 \_2$ & 418 & 0 & 418 & 81 & 16.37 & 0.14 & $180 / 106$ \\
\hline 18 & 329 & 174 & 155 & 42 & 11.25 & 5.08 & $146 / 93$ \\
\hline 19 & 923 & 282 & 641 & 125 & 15.25 & 2.19 & $146 / 59$ \\
\hline 20_1 & 711 & 236 & 475 & 92 & 28.86 & 0.66 & $146 / 67$ \\
\hline $20 \_2$ & 377 & 20 & 357 & 69 & 32.51 & \begin{tabular}{|l|}
0.98 \\
\end{tabular} & $160 / 93$ \\
\hline $20 \_3$ & 460 & 27 & 433 & 84 & 22.26 & 0.57 & $166 / 93$ \\
\hline 21 & 689 & 10 & 679 & 132 & 10.76 & 2.98 & $166 / 105$ \\
\hline 22 & 570 & 360 & 210 & 41 & \begin{tabular}{|l|}
15.19 \\
\end{tabular} & 1.33 & $186 / 106$ \\
\hline 23 & 545 & 205 & 340 & 66 & \begin{tabular}{|l|}
13.28 \\
\end{tabular} & 1.73 & $160 / 93$ \\
\hline 24 & 834 & 180 & 654 & 127 & 30.46 & 2.64 & $213 / 128$ \\
\hline 25 & 402 & 12 & 390 & 76 & \begin{tabular}{|l|}
38.13 \\
\end{tabular} & $\begin{array}{ll}1.38 \\
\end{array}$ & $160 / 80$ \\
\hline 26 & 875 & 172 & 703 & 137 & 22.82 & 2.16 & $186 / 106$ \\
\hline 27 & 688 & 207 & 481 & 94 & 3.82 & 1.27 & $173 / 93$ \\
\hline
\end{tabular}

Table 2. ICP: intracranial pressure.

\section{Results}

The data were measured at one time in 18 patients, two times in eight patients, three times in three patients and four times in two patients. A total of 43 data were available from 27 patients and the total measured time was 29,653 seconds (measured time in each data: min. 256, max. 2,562, mean: $690 \mathrm{sec}$.). The total valid data time (measured time - reject time) was 19,256 seconds (valid time in each data: min. 102, max. 872, mean: 470 sec.). The total number of columns was 3,800 (number of columns in each data: min. 20, max. 170, mean: 93). The measured mean ICP value in each data was from 3.82 to 69.39 (mean: 25.9) hPa (Tables 2 and 3).

The ICP value and the NRF of the brain from 41 data were plotted in Fig. 4. 


\begin{tabular}{|c|c|c|c|c|c|c|}
\hline \multirow{3}{*}{$\begin{array}{l}\text { Data } \\
\text { number }\end{array}$} & \multicolumn{6}{|l|}{ Caliculated results } \\
\hline & \multirow{2}{*}{\begin{tabular}{|l|} 
Number of poles \\
(np)
\end{tabular}} & \multirow{2}{*}{\begin{tabular}{|l|} 
Number of zeros \\
(zp)
\end{tabular}} & \multirow[b]{2}{*}{ Delay } & \multirow[b]{2}{*}{$\mathbf{Q}$} & \multirow{2}{*}{$\begin{array}{l}\text { \%fit } \\
(\%)\end{array}$} & \multirow{2}{*}{\begin{tabular}{|l|} 
Calcurated NRF \\
$(\mathrm{Hz})$
\end{tabular}} \\
\hline & & & & & & \\
\hline 1 & 6 & 4 & 8 & 0.97 & 92.95 & 30.20 \\
\hline $2 \_1$ & 8 & 6 & 4 & 2.91 & 90.34 & 15.85 \\
\hline $2 \_2$ & 6 & 1 & 3 & 6.33 & 83.38 & 23.55 \\
\hline $3 \_1$ & 6 & 5 & 6 & 1.67 & 64.63 & 31.53 \\
\hline $3 \_2$ & 7 & 1 & 0 & 4.94 & 61.15 & 20.29 \\
\hline $4 \_1$ & 6 & 1 & 4 & 5.44 & 64.64 & 19.47 \\
\hline $4 \_2$ & 8 & 3 & 7 & 0.75 & 73.99 & 19.74 \\
\hline 5 & 8 & 1 & 1 & 3.03 & 67.63 & 23.02 \\
\hline $6 \_1$ & 8 & 7 & 2 & 6.50 & 85.92 & 33.15 \\
\hline $6 \_2$ & 7 & 2 & 5 & 4.28 & 82.44 & 33.55 \\
\hline $6 \_3$ & 5 & 1 & 3 & 3.75 & 83.95 & 16.56 \\
\hline 7_1 & 7 & 3 & 2 & 5.24 & 62.37 & 36.48 \\
\hline 7_2 & 6 & 2 & 4 & 1.92 & 64.75 & 36.44 \\
\hline 7_3 & 7 & 5 & 4 & 2.71 & 61.35 & 29.87 \\
\hline 7_4 & 7 & 2 & 7 & 5.17 & 64.51 & 35.10 \\
\hline $8 \_1$ & 6 & 3 & 6 & 5.08 & 62.56 & 32.25 \\
\hline $8 \_2$ & 7 & 3 & 0 & 0.61 & 75.59 & 31.40 \\
\hline $8 \_3$ & 5 & 1 & 5 & 3.32 & \begin{tabular}{|l|}
63.8 \\
\end{tabular} & 42.51 \\
\hline 9 & 5 & 3 & 7 & 2.36 & 61.57 & 13.80 \\
\hline 10 & 4 & 3 & 3 & 2.00 & 61.77 & 18.27 \\
\hline 11 & 7 & 6 & 2 & 1.51 & \begin{tabular}{|l|}
88.78 \\
\end{tabular} & 19.22 \\
\hline 12 & 7 & 3 & 3 & 3.08 & 62.46 & 15.42 \\
\hline 13 & 7 & 5 & 6 & 2.05 & 68.17 & 24.75 \\
\hline 14 & 7 & 6 & 2 & 3.71 & 87.47 & 45.38 \\
\hline 15 & 7 & 1 & 1 & 4.07 & 76.54 & 20.97 \\
\hline $16 \_1$ & 6 & 2 & 6 & 3.20 & 81.54 & 35.61 \\
\hline $16 \_2$ & 6 & 3 & 8 & 3.67 & 82.11 & 30.88 \\
\hline $16 \_3$ & 7 & 4 & 8 & 4.37 & 83.11 & 36.56 \\
\hline $16 \_4$ & 6 & 5 & 3 & 1.62 & 84.47 & 19.79 \\
\hline $17 \_1$ & 5 & 3 & 0 & 1.51 & 63.83 & 23.08 \\
\hline $17 \_2$ & 7 & 4 & 8 & 2.89 & 62 & 22.11 \\
\hline 18 & 7 & 3 & 2 & 2.03 & 76.69 & 17.27 \\
\hline 19 & 5 & 4 & 6 & 0.84 & 88.31 & 20.17 \\
\hline $20 \_1$ & 8 & 3 & 7 & 3.74 & 84.94 & 28.94 \\
\hline $20 \_2$ & 6 & 2 & 3 & 1.46 & 91.63 & 30.35 \\
\hline $20 \_3$ & 7 & 2 & 8 & 3.23 & \begin{tabular}{|l|}
89.09 \\
\end{tabular} & 24.80 \\
\hline 21 & 7 & 3 & 0 & 1.38 & \begin{tabular}{|l|}
77.37 \\
\end{tabular} & 17.90 \\
\hline 22 & 6 & 5 & 6 & 3.09 & 71.66 & 20.80 \\
\hline 23 & 6 & 5 & 2 & 1.65 & \begin{tabular}{|l|}
79.97 \\
\end{tabular} & 19.00 \\
\hline 24 & 5 & 1 & 1 & \begin{tabular}{|l|}
5.87 \\
\end{tabular} & \begin{tabular}{|l|l|}
67.45 \\
\end{tabular} & 29.59 \\
\hline 25 & 6 & 2 & 1 & 1.01 & \begin{tabular}{|l|}
63.08 \\
\end{tabular} & 33.09 \\
\hline 26 & 3 & 2 & 2 & 2.57 & \begin{tabular}{|l|}
76.3 \\
\end{tabular} & 26.47 \\
\hline 27 & 8 & 1 & 0 & 1.25 & \begin{tabular}{|l|}
76.6 \\
\end{tabular} & \begin{tabular}{|l|}
10.71 \\
\end{tabular} \\
\hline
\end{tabular}

Table 3. NRF: natural resonance frequency.

Every data was presented on the quadratic functions graph $\left(\mathrm{ICP}=0.0329(\mathrm{NRF})^{2}+0.0842 \mathrm{NRF}\right)$. These parameters strongly correlated with each other $\left(R^{2}=0.9952\right.$ coefficient of determination).

\section{Discussion}

Many individual parameters may contribute to the NRF of the brain, such as intracranial volume, arterial wall stiffness, age, disease, blood pressure, blood concentration, cardiac output, general peripheral vessel resistance and body position. Furthermore, fluctuation of CCPW, ICP value and PWICP in every cardiac pulse makes it difficult to analyse. The respiratory rhythm is one of the most significant factors to modulate the ICP value, in which the modulation ratio of PWICP power spectral is over $55 \%{ }^{26}$. The intrathoracic pressure decreases in exhalation and increases in inspiration. The jugular venous pressure is dependent on the intrathoracic pressure and the 


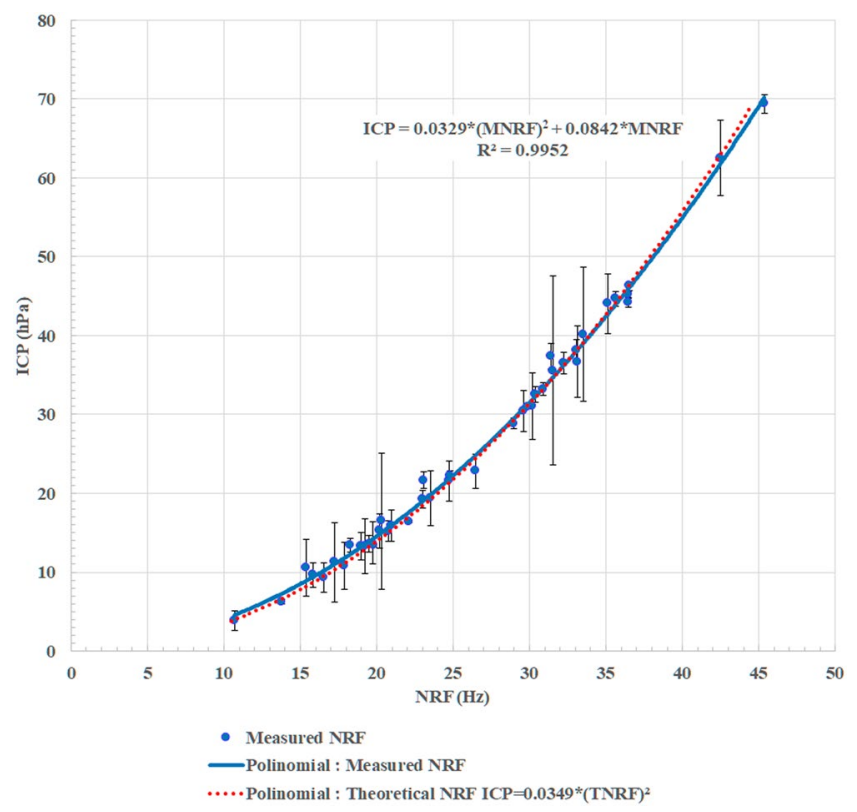

Figure 4. The graph for the relationship between the ICP value and the NRF of the brain. Blue points indicate data number $(n=40)$. Error bars indicate SD. These parameters are strongly correlated with each other $(\mathrm{R} 2=0.9998)$. The blue polynomial: approximation graph from blue points. The red polynomial: theoretical calculation graph from discussion.

intracranial pressure is depending on the jugular venous pressure. We utilised the weight averaging method for respiratory synchronization in which the largest amplitude of CCPW in the 10.12 second column was picked-up. By the averaged CCPW and PWICP without respiratory modulation, the calculated NRF of the brain and measured ICP value were presented on the quadratic functions graph. This means that individual parameters without respiratory modulation are not related to the NRF determination.

Theoretical analysis for NRF of the brain. This relationship between the ICP value and the NRF of the brain can be explained by the simple brain acoustic dynamics model (Fig. 5 left). The brain (weight: $m \mathrm{~kg}$ ) is located in the closed space and the CSF interferes with the movement of the brain by the effect of a shock absorber (cerebrospinal volumetric compliance: $C F$ ) and viscosity (resistance: $R \Omega$ ). The arterial pulse (force: $F \mathrm{~N}$ ) moves the brain. The moving speed of the brain is velocity: $V \mathrm{~m} / \mathrm{s}$. Each factor can be described as:

$$
f=R v+m \frac{d v}{d t}+\frac{1}{C} \int v d t
$$

These components can be changed in the electric circuit (Fig. 5 right).

If the arterial pulse can be substituted by a sigmoid curve, the NRF of the brain: $f_{0}(\mathrm{~Hz})$ can be described as:

$$
\mathrm{f}_{0}=\frac{1}{2 \pi \sqrt{m C}}
$$

By this theory, the NRF of the brain is only dependent on brain weight and cerebrospinal volumetric compliance. The average brain weight is $1.4 \mathrm{~kg}^{27}$. Cerebrospinal volumetric compliance is inversely proportional to the ICP value $e^{25,28,29}$. When the ICP is $13.33 \mathrm{hPa}$, the compliance was $0.0000473^{30}$; the coefficient: $C$ of the cerebrospinal compliance can be calculated by:

$$
C=\frac{0.0000473}{\left(\frac{I C P}{13.33}\right)}
$$

and the theoretical calculated relationship between ICP and the NRF is described as shown in Fig. 3 (red dots). Although the graph was introduced by substituting the previous proposed data into the simple brain mechanical model, our clinical data was strongly correlated $\left(\mathrm{R}^{2}=0.9999\right.$ coefficient of determination and the standard error between the polynomial curves is $0.55 \mathrm{hPa}$ ). The strong correlation between the ICP value and the NRF of the brain from individual data means that the ICP value can be predicted from the NRF of the brain.

Limitation. To understand the details of the relationship between the ICP value and the NRF of the brain, further data collection is necessary. Especially data for high ICP values, which is lacking in our research. Because increased ICP value remarkably decreases inflow of the blood to the intracranial space, the drastic change around the "non-filling" situation occurs. The adaptable range of cerebral autoregulation is different in each patient. Our 

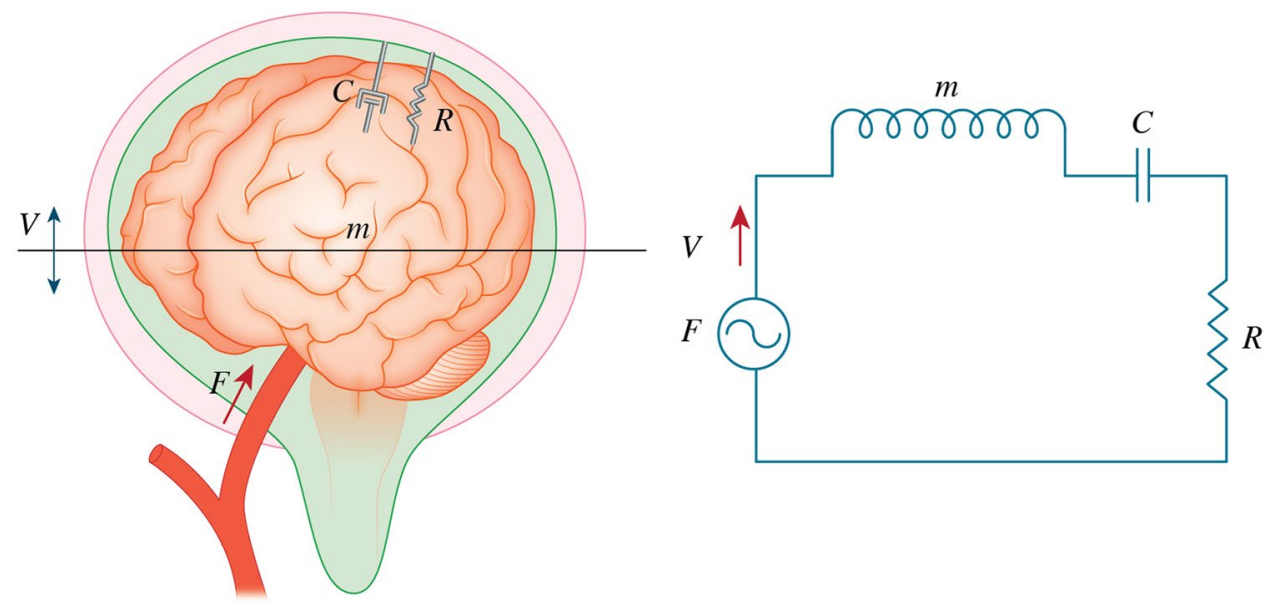

Figure 5. Theoretical analysis of the resonance phenomenon in the skull. Left: the schema of the simple brain acoustic dynamics model. The brain (weight: $\mathrm{m} \mathrm{kg}$ ) is located in the closed space. The CSF interferes with the movement of the brain by the effect of a shock absorber (cerebrospinal volumetric compliance: $\mathrm{C} \mathrm{F}$ ) and viscosity (resistance: $R \Omega$ ). The arterial pulse (force: $\mathrm{fN}$ ) moves the brain. The moving speed of the brain is velocity: v m/s. Right: mechanodynamically electrically equivalent circuit (weight: $\mathrm{m} \mathrm{kg}$ ) (condenser: $\mathrm{C} \mathrm{F}$ ) (resistance: $\mathrm{R} \Omega$ ) (force: $\mathrm{f} \mathrm{N}$ ).

result cannot adjust in very high range of ICP patient. We have no data on the small brain weights of paediatric and senile patients. External decompression surgery might affect our results. Radiological evaluation is also necessary.

Future perspective. The correlation between the ICP value and the NRF of the brain may be utilised in the prediction of the ICP value. If the NRF of the brain can be recorded from somewhere, we can predict the ICP value. Theoretically the NRF can be transmitted and measured from various places, such as the movement of the tympanum, external ear pressure waveform and the thickness of the retinal membrane. Of course, these data must include not only the NRF of the brain, but also other resonance and oscillation frequency from other organs. If we can extract this specific frequency from extracranial organs, non-invasive intracranial pressure prediction will be possible.

\section{Conclusions}

The individual NRF, which was calculated by the transfer function method using their CCPV and PWICP, only depends on the measured ICP value from ventricular drainage or an ICP sensor, based on the simple quadratic functions as presented. This means that individual parameters, without respiratory modulation, are not related to the NRF determination. This relationship could be explained by a simple brain mechanical model. The ICP value might be predicted by some non-invasive methods using this principle because the NRF consists of relative value.

Received: 12 July 2019; Accepted: 28 January 2020;

Published online: 13 February 2020

\section{References}

1. Czosnyka, M. Monitoring and interpretation of intracranial pressure. J. Neurol. Neurosur. Psychiatry. 75, 813-821 (2004).

2. Zhang, X. et al. Invasive and noninvasive means of measuring intracranial pressure: A review. Physiol. Meas. 38, 143-182 (2017).

3. Bhatia, A. \& Gupta, A. K. Neuromonitoring in the intensive care unit. I. Intracranial pressure and cerebral blood flow monitoring. Intensive Care Med. 33, 1263-1271 (2007).

4. Wakerley, B. R. et al. Usefulness of transcranial doppler derived cerebral hemodynamic parameters in the noninvasive assessment of intracranial pressure. J. Neuroimaging. 25, 111-116 (2015).

5. Gwer, S. et al. The tympanic membrane displacement analyser for monitoring intracranial pressure in children. Childs Nerv. Syst. 29, 927-933 (2013).

6. Evensen, K. B., Paulat, K., Prieur, F., Holm, S. \& Eide, P. K. Utility of the Tympanic membrane pressure qaveform for non-invasive estimation of the intracranial pressure waveform. Sci. Rep. 25, 15776, https://doi.org/10.1038/s41598-018-34083-6 (2018).

7. Maissan, I. M. et al. Ultrasonographic measured optic nerve sheath diameter as an accurate and quick monitor for changes in intracranial pressure. J. Neurosurg. 123, 743-747 (2015).

8. Oliver, G., Stylianos, M., Andreas, S., Björn, S. \& Rudolf, R. Evaluation of a novel noninvasive ICP monitoring device in patients undergoing invasive ICP monitoring: preliminary results. J. Neurosurg. 128, 1653-1660 (2018).

9. Nair, S. Clinical review of non-invasive intracranial pressure measurement in medical cases. J. Neuroanaesthesiology Crit. Care. 3, 9-14 (2016).

10. Khan, M. N., Shallwani, H., Khan, M. U. \& Shamim, M. S. Noninvasive monitoring intracranial pressure - a review of available modalities. Surg. Neurol. Int. 51, 1-11 (2017).

11. Raboel, P. H., Bartek, J. Jr., Andresen, M., Bellander, B. M. \& Romner, B. Intracranial pressure monitoring: invasive versus noninvasive methods-a review. Crit. Care Res. Pract. 2012, 950393, https://doi.org/10.1155/2012/950393 (2012).

12. Furihata, K. \& Yamashita, M. Transfer function for vital infrasound pressure between the carotid artery and the tympanic membrane. J. Acoust. Soc. Am. 133, 1169-1186 (2013). 
13. Bhimani, S., Virapongse, C. M. \& Sarwar, M. High-resolution computed tomographic appearance of normal cochlear aqueduct. AJNR. 5, 715-720 (1984).

14. Matsuyama, M. Recording of the effects on the external auditory canal pressure waves of intracranial pressure pulse waves and blood pressure waves (in Japanese). J. Keio Med. Society. 72, 497-509 (1995).

15. Marchbanchs, R. J., Reid, A., Martin, A. M., Brightwell, A. P. \& Bateman, D. The effect of raised intracranial pressure on intracochlear fluid pressure: three case studies. Br. J. Audiol. 21, 127-130 (1987).

16. Lang, E. W., Paulat, K., Witte, C., Zolondz, J. \& Mehdorn, H. M. Noninvasive intracranial compliance monitoring. J. Neurosurg. 98, 214-218 (2003)

17. Hirsch, A.E., Ommaya, A.K. \& Mahone, R.M. Tolerance of subhuman primate brain to cerebral concussion. Washington, DC: Naval Ship Research and Development Center. 2876 (1968).

18. Anile, C., Portnoy, H. D. \& Branch, C. Intracranial compliance is time-dependent. Neurosurgery. 20, 389-395 (1987).

19. Kasuga, Y., Hasegawa, Y., Nitta, M. \& Nagai, H. Systems analysis of pressure transmission in intracranial cavity (in Japanese). No Shinkei 36, 1015-1023 (1984).

20. Kasuga, Y., Nagai, H., Hasegawa, Y. \& Nitta, M. Transmission characteristics of pulse waves in the intracranial cavity of dogs. J. Neurosurg. 66, 907-914 (1987).

21. Takizawa, H., Gabra-Sanders, T. \& Miller, J. D. Changes in the cerebrospinal fluid pulse wave spectrum associated with raised intracranial pressure. Neurosurgery. 20, 355-361 (1987).

22. Michaeli, D. \& Rappaport, Z. H. Tissue resonance analysis; a novel method for noninvasive monitoring of intracranial pressure. Technical note. J. Neurosurg. 961, 132-1137 (2002).

23. Gupta, R. K. \& Przekwas, A. Mathematical models of blast-induced TBI: current status, challenges, and prospects. Front. NEUROLOGY. 59, 1-21 (2013).

24. Laksari, K., Wu, L. C., Kurt, M., Kuo, C. \& Camarillo, D. C. Resonance of human brain under head acceleration. J. R. Soc. Interface. 12, 1-9 (2015).

25. Avezaat, C. J., van Eijndhoven, J. H. \& Wyper, D. J. Cerebrospinal fluid pulse pressure and intracranial volume-pressure relationships. J. Neurology, Neurosurgery, Psychiatry. 42, 687-700 (1979).

26. Strik, C., Klose, U., Erb, M., Strik, H. \& Grodd, W. Intracranial oscillations of cerebrospinal fluid and blood flows: analysis with magnetic resonance imaging. J. Magnetic Reson. Imaging. 15, 251-258 (2002).

27. Kopsch, F. R. Lehrbuch und Atlas der anatomie des menschen band III nervensystem-sinnesorgane (ed. Kopsch, F. R.) 44-49 (Georg Theme Leipzig 1953)

28. Lange, S.A., Vlieger, M., Go, K.G. \& Stassen, H.G. Cerebrospinal fluid pulse pressure and craniospinal dynamics (ed. Avezaat, C. J. \& van Eijndhoven, J. H.) 210-216 (The Hague Jongbloed en Zoon 1984).

29. Marmarou, A., Shulman, K. \& LaMorgese, J. Compartmental analysis of compliance and outflow resistance of the cerebrospinal fluid system. J. Neurosurg. 43, 523-534 (1975).

30. Martin, B. A., Reymond, P., Novy, J., Baledent, O. \& Steriopulos, N. A coupled hydrodynamic model of the cardiovascular and cerebrospinal fluid system. Am. J. Physiol. Heart. Circ. Physiol. 302, 1492-1509 (2012).

\section{Acknowledgements}

Special thanks to Mr. Norio Koike and Mr. Satoshi Yasumoto for manufacturing original measurement and recording apparatus in Ichikawa Electric Corporation Ltd. Tokyo, Japan. This research was funded by Japan Science and Technology Agency (JST). A-STEP feasibility study in 2012, and by Japan's New Energy and Industrial Technology Development Organization (NEDO) in 2013, and by Grants-in-Aid for scientific research in Ministry of Education, Culture, Sports, Science and Technology-Japan from 2015 to 2017 and 2018 to 2020. Receiver: Kenji Furihata.

\section{Author contributions}

K.H. and T.G. conceived the project, managed patients and investigated clinical data. K.F. and T.G. designed the study. K.F. designed measurement apparatus, constructed the mathematical model and analysed the data. T.G. and K.F. wrote the paper and K.H. supervised the paper. The manuscript reflects the contributions of all authors.

\section{Competing interests}

The authors report no financial or non financial competing interests. Although we have some patents for prediction of intracranial pressure, all of the claims belong to affiliated university not to individual. We have no personal financial interest in this paper.

\section{Additional information}

Correspondence and requests for materials should be addressed to K.F.

Reprints and permissions information is available at www.nature.com/reprints.

Publisher's note Springer Nature remains neutral with regard to jurisdictional claims in published maps and institutional affiliations.

Open Access This article is licensed under a Creative Commons Attribution 4.0 International License, which permits use, sharing, adaptation, distribution and reproduction in any medium or format, as long as you give appropriate credit to the original author(s) and the source, provide a link to the Creative Commons license, and indicate if changes were made. The images or other third party material in this article are included in the article's Creative Commons license, unless indicated otherwise in a credit line to the material. If material is not included in the article's Creative Commons license and your intended use is not permitted by statutory regulation or exceeds the permitted use, you will need to obtain permission directly from the copyright holder. To view a copy of this license, visit http://creativecommons.org/licenses/by/4.0/.

(c) The Author(s) 2020 